\title{
Entropy: First as Chaos then as Order
}

\begin{abstract}
This article presents a reformulation to the concept of Entropy and the Second Law of Thermodynamics. Research shows that the second law of Thermodynamics is misunderstood, by changing the Law and re-defining Entropy, the improved statement of the law is the fundamental law that drives our Universe, following Hegel's oeuvre.
\end{abstract}

Keywords: Order· Loschmidt's paradox · Entropy· Hegel· Lacan

\section{Introduction}

Apropos of the aphorism first as tragedy, then as farce defining entropy as a measure of chaos and stating that it increases with time -Second law of Thermodynamics - was a tragedy due to which we grasped the clouds of error instead of heavens of truth -in Hegelian terms. It is the humble aim of the article to remedy the error and in doing so; parallelism with Hegel's philosophy is made. First, let's uncover history.

E.D. Schneider and J.J. Kay [1] tried to explain many naturally occurring phenomena using an extended Second Law. Ben-Naim [2] showed that existing definitions of Entropy and Second law are fallacious. J.B Brissaud [3] acknowledges that entropy, more than any other physical quantity has led to various interpretations, Shannon [4] identifies it with positive information, Boltzmann with the 'disorder', Brillouin [5] [6] with lack of information or ignorance.

J. Neumann famously [7] said, "No one really understands entropy". Understanding of thermodynamic entropy has been an open question in the philosophy of physics and it is safe to say that we have not reached a scientific consensus on the understanding of entropy and the second law and this paper is the much-needed revision, after all, it was Hegel who taught - permanent 
revisionism. Since Clausius's formulation, the definition has been critiqued by many and now it is associated with information entropy along with few others. This paper aims to reformulate the thermodynamic entropy and the second law as proposed by Clausius to explain the pattern observed by him in natural phenomena. Let's revisit the criticism of second law and definition of entropy then proceed towards re-defining both the terms and it is shown that proposed law is a very Hegelian concept.

Carlo Rovelli [8] [9] propounds the need to incorporate philosophy in physics and discusses ancient philosopher such as Anaximander, Hecateaeus, Democritus, Plato and Aristotle but even when positing - albeit naively - Hegelian ideas such as:

- The World is nothing but change.

- The best grammar for thinking about the world is of change, not of permanence. Not of being, but of becoming.

- We are histories of ourselves.

- It's not energy that the world needs rather its low entropy.

Never mentions the Hegel who, above all is the philosopher of change.

\section{Critique of The Second law of thermodynamics.}

The Second Law of Thermodynamics and Entropy is one of the most mysterious concepts in physics. There are various "formulations" of the second law of thermodynamics and most of them have been criticized by Arieh Ben-Naim [10][11]. Let's revisit the critique.

\subsection{Clausius definition}

The entropy of the universe always increases. 
This formulation is found in most Science textbooks but this quite poor definition as the entropy of the universe, as criticized is a meaningless statement. Authors like Atkins define thermodynamic entropy as "a measure of chaos" and state entropy always increases as chaos always increases without ever defining "chaos". Traditionally, there have been many manifestations of Second law: "Entropy always increases", As pointed out by Ben Naim, "many authors often spend a lot of time discussing the manifestations of the second law of thermodynamics but very little time discussing what is common between those manifestations". A few manifestations like; water always flows downhill, two gases spontaneously mix, heat always flows from a body at a high temperature(hence is cooled) to a body at a lower temperature (which is heated up) show that state of all the system arrives at a stable state after some time. Of course, the bland truism, a stable state is a state of the system when state variables stop varying, in context with which the change was being observed and State of every system proceeds towards the most probable state or stable state is a fundamental law from probability theory.

Two problems that affect our understanding of the law are:-

1. Misinterpreting or not defining order and chaos in this framework.

2. We know that the state of every system proceeds towards a stable state after some time. Hence, confusing this fact with the statement, "entropy always increases".

\section{Proposed Law}

Let's define System, State function and Entropy then proceed towards the statement of the law proceeding heuristically. 


\subsection{System}

Posit: A system in space is a boundary which contains other system and is enclosed by another system. Therefore, each system (2) encloses another system (1), and another system (3) encloses the system (2).

Example: Pendulum (say, system 2) is enclosed by its surrounding air (system3) and it encloses material of the ball and string (system 1).

\subsection{State Function}

Posit: State function is a function of state variables.

That is a function relating information about parameters that we are interested in studying. Some examples of state variables are displacement, pressure, volume, number of molecules. One must carefully choose state variables depending on the purpose.

\subsection{Hegel and Entropy}

Quotations - to prevent bastardization- from Solomon [12] shall be used as a presupposition to posit analytically:

Presupposition 3.3.1: "What is that point? In one sense, it is simply the point that all concepts are context-bound, "the fluidity of the Concept." But in a larger sense, the point is that philosophical terms and statements are essentially interrelated; they receive their meaning and their plausibility (or lack thereof) only by virtue of other terms and statements, against a background of other claims and principles, within the history of a discipline which has developed a certain way of looking at the world and certain terms to express it. Indeed, the main point of the Phenomenology, which we will 
examine in the following sections, is just this historical unity of conceptual thought, the "dialectical movement" which holds our shared consciousness of the world together even as it pulls it apart with differences and conflicts of opinion".

Presupposition 3.3.2: “Hegel's original intentions for the book, is what might be called historicism, the view that various "forms of consciousness" are relative to a time and a viewpoint".

Presupposition 3.3.3: "This idea that various "forms of consciousness" are relative to time and a viewpoint".

\subsection{Entropy}

Posit 3.4.1: Change in Entropy is the change of state function with respect to a quantity that measures the change from the stable state of the system.

Hence, Change in Entropy is equivalent to "forms of consciousness" or "the fluidity of the Concept" and the quantity that measures the change is the "viewpoint" and change is measured from the stable state since the viewpoint in itself cannot be too distorted as it will prevent proper measurement.

Let,

\section{$d S:$ Change in entropy}

$\varphi$ : State function to describe the state of the system (using state variables) with respect to a Stable State.

$t$ : Quantity used to measure changes in state variables from the stable state, usually, Time or Temperature but is not limited to only these two quantities, it is, in general, a quantity that measures the change. 


$$
d S=d \varphi / d t
$$

Comparing with presupposition (3.3.3): This idea that various "forms of consciousness" -State function- are relative to time and a viewpoint is what is perfectly encapsulated by the proposed definition of entropy.

Furthermore, a similar emphasis on "the Concept" can be found in reformulations of Relativity and Quantum mechanics by Aerts, D $[13,14]$ as,"... And according to the conceptuality interpretation, what has always been in plain sight, but precisely for that was very hard to notice, is that the notion one should use to represent the nature of a quantum entity and make full sense of its behaviour is the very notion of concept!"

\subsection{Hegel and Second law}

Hegel's phenomenology [15] is more about changes in experience and changes in the forms of experience and transformation between concepts rather than just static experiences, these, move in the direction of making sense of things in a unified manner that is not just Hegel's principle but the most intrinsic desire of all human experience.

Quotations from Solomon [12] will be used as pre-supposition to posit homology with the proposed law.

\section{Presupposition 4.1.1:}

- "Hegel is no mere phenomenologist, providing us with a detailed description or an encyclopaedia of conceptual forms; he is also a dialectician, for whom the transitions between those forms are at least as important as the forms themselves". 


\section{Presupposition 4.1.2:}

- "What Hegel is doing in the Phenomenology is presenting the development of the forms of human experience, i.e. "the Concept," as a sequence, in which the examples need not be in anything resembling historical order but nevertheless represent a sequence which can be found in time, if not invariably. It is a sequence which moves mainly by "opposites," and its general direction is the direction of increasing generality".

\subsection{Proposed statement of the second law}

Productive Force [Kraft] is mind striving to organize itself; so too in the outer world, a universal tendency to organize must reveal itself.

-F.W.J. von Schelling, Ideas towards a Philosophy of Nature

Posit: The state of a system (1) changes from unstable states to a stable state and will release its entropy to another system (2) until it is at a stable state.

The idea in presupposition (4.1.3), that "forms of Consciousness" - Entropy, is a sequence which moves mainly by "opposites" (unstable states) and its general direction is the direction of increasing generality (Order or towards Stable state) is perfectly captured by the posit (4.2.1).

The proposed law is about the way a given form (system) changes which is akin, Hegel who above all is the "philosopher of change".

The decrease in entropy is observed because the change in state variables de-creases as the state of the system is approaching the stable state. Recall, the stable state is the state of the system when the state variables stop changing (in context with which change was being observed) therefore, entropy is bound to decrease to go at a state when it stops changing. According to this, the entropy of a system can both increase and decrease. Increase in entropy is observed when entropy is added 
to the system and state of the system temporarily goes to an unstable state and decrease is observed when the idle system is approaching the stable state.

\subsubsection{Example: Motion of the Pendulum}

We will measure the displacement of the pendulum from the original stable position to study the motion of a pendulum.

$$
\text { State variable }(\varphi)=\text { Displacement }
$$

Time: the quantity which measures the change of state variable from the stable state.

Hence,

$$
\begin{gathered}
d S=d x / d t \\
d S=\text { velocity }
\end{gathered}
$$

The Pendulum acquires entropy by a gentle push. It will then show damped oscillations around a stable state continuously releasing acquired entropy to another system. Therefore, Entropy decreases and becomes constant equilibrium state, it could be pointed out that negative of decreasing quantity is increasing quantity but the point is definition presented in this article is much more intuitive than existing definitions.

\subsubsection{Example: Transfer of Heat}

If the system has a temperature $T$ and a small amount of heat $d Q$ is given to the system, we define the change in entropy as

$$
d S=d Q / T
$$


This is, change in heat energy with respect to a quantity which measures the heat energy with respect to a quantity that measures average heat energy, as Temperature is simply a measure of average thermal energy. Initially, the heat will flow from the body at a high temperature to a body at a lesser temperature. Then, after a period of time, the temperature of the system will become uniform and reach a state called thermal equilibrium just as predicted by the proposed law.

\subsubsection{Newtonian Mechanics and Second Law}

The definition of Entropy and the Second law is the most fundamental law that drives our Universe. Here, it is shown that Newton's First Law is a manifestation of proposed Second law.

Bowman [16] acknowledged, "In Newtonian Mechanics, the state of the system at a particular time is given by positions and velocities of all its constituent parts. From this information (position and velocity are essential information about the state of the system, hence entropy) we use Newton's Laws to determine the state of the system at a given time".

Taking a system whose state variable is velocity. In such a case when entropy is added to the system the velocity of the particle will increase and when entropy is not added the velocity will proceed to a stable state (In real systems there is always friction so it will slow down, therefore the particle will proceed towards the stable state or if there is no friction even then it will be at the stable state).

State variable $(\phi)=$ velocity

Time is a quantity which measures the change of the state function from the stable state.

Hence,

$$
d S=d v / d t
$$

Therefore, 


$$
d S=\text { acceleration }
$$

This is essentially what Newton's law states, that, the system stays in a stable state unless some force is applied to the system.

\section{Order and Chaos}

Despite the subjectivity in the conception of terms Order and Chaos, they can be objectively understood in this framework, as follows:-

Imagine a flask sitting on a table. Due to wind the flask falls and breaks into pieces. Most people are tempted to say, "See! The system is now more chaotic than the initial state as there are more pieces scattered around and is not as ordered as the flask, as a whole was, initially". This bias towards labelling the final state of the system more chaotic seems aesthetical. Let's, analyse the event through the eyes of the proposed framework. Initially, system-1 contains only the flask. Due to the wind (Imagine as a form of entropy added to the system-1 from another system-2) now the system goes through various unstable states in air and is constantly acted by 'gravitational' force and finally hits the ground shattering into pieces. Therefore, reaching the most probable or stable state. To conclude, now nothing naturally can happen further (unless some other form of entropy is added to the system), the system is finally in most probable state or stable state. Therefore, labelling the stable state as a state of order and states in which the flask was in-mid-air as the state of chaos makes more sense. In this way, the system always proceeds to the ordered state. 


\section{Entropy at Equilibrium - The Absolute}

At the stable state, the system reaches the equilibrium. That is a state which does not change with time. Let's, further the investigation via Planck's reformulation of Boltzmann's equation and Boltzmann, Zermelo debate.

\subsection{Boltzmann's Entropy}

The initial state in most cases is bound to be highly improbable and from it, the system will always rapidly approach a more probable state until it finally reaches the most probable state, i.e. that of heat equilibrium.

-Ludwig Boltzmann

The proposed law is similar to Boltzmann's definition of entropy and second law, but there is a significant difference. That is this definition must not be limited to heat and thermodynamics (thermal equilibrium) but it should be universal. Boltzmann explicitly stated, “. . a system ... when left to itself, it rapidly proceeds to disordered, most probable state." This is where the proposed framework is novel and differs from Boltzmann's work. As concluded previously, that labelling most probable state as the state of order is more sensible.

\subsubsection{On the Mathematical statement}

The first proper mathematical derivation of the second law was given by Boltzmann via H-theorem. This was refuted by Ernst Zermelo [17] via Poincare's recurrence theorem as follows:

Theorem 6.1 (Recurrence theorem):

Let $\left\langle\Gamma, \aleph, \mu,\left\{\mathrm{T}_{\mathrm{t}}\right\}\right\rangle$ be any dynamical system such that $\mu(\Gamma)<\infty$, and let $A \in \mathfrak{\aleph}$ be any measurable set in $\Gamma$. Consider any $\tau>0$ and let 


$$
B=\left\{x \in \Gamma: x \in A \quad \& \forall t>\tau\left(T_{t} x \notin A\right)\right\},
$$

the set of states in $A$ that after time $\tau$ have left $A$ and will never return to $A$. Then

$$
\mu(B)=0
$$

Here, a dynamical system $\left\langle\Gamma, \aleph, \mu,\left\{T_{t}\right\}\right\rangle$ consists of a measure space $\langle\Gamma, \aleph, \mu\rangle$ in the sense of measure theory. To turn a measure space into a dynamical system, one further assumes the existence of a one-parameter group of measurable evolution operators $\mathrm{T}_{\mathrm{t}}: \Gamma \rightarrow \Gamma$ for all $t \in \mathbb{R}$ which has the group property $T_{t} \circ T_{t}=T_{t+t}$

and is measure-preserving:

$$
\forall t \in \mathbb{R}, \forall A \in \aleph: \mu\left(T_{t} A\right)=\mu(A)
$$

It is a consensus that he won the battle but fell short in giving an acceptable formulation of second law which was homogenous.

The Boltzmann equation in mathematical form was paradoxically never given by him rather it was Planck $[18,19]$ who defined equilibrium entropy in its mathematical form. The explanation proposed by Planck rests on the observation that, due to the identity of the atoms, two complexions differing only by a permutation of the coordinates of the latter represent the same physical state. That is when the co-ordinates change but the physical state of the system remains the same by returning to itself and tries to preserve its identity. Even Lacan [20] said," For, what is a thing, if not that which endures, in an identical state, for a certain time?"

The author acknowledges his limited mathematical skills - in the spirit of Ludwig Wittgenstein - who famously said," Whereof one cannot speak, thereof one must be silent". Although, speculatively the 
mathematical form must be as Zizek [21] says," Hegelian Absolute Knowledge - the closed 'circle of circles' “. This movement of circles must depend on frequency and wavelength.

\section{Arrow of Time and Loschmidt's paradox}

Most, perceive Newtonian mechanics as reversible mechanics, this is statement is reproachable, since, the flow of time is an 'a priori' or a presupposition - on which the entire framework is based. This apoplectic phenomenon of irreversibility was observed when formulating entropy. As elucidated by Jerome Rothstein [22]:

- "It is fair to say that irreversibility does not occur in the discussion, for the simple reason that mention of it has been suppressed".

Few authors like Ben Naim do not believe in the arrow of time which is associated with the second law. Let's go through an example from Ben Naim's book [7] which indicates that arrow of time does exist i.e., over a period of time, state of the system proceeds towards the more probable state or stable state and irreversibility exist due to formation of order, unlike the traditional arrow of time which blandly points towards increasing entropy. Let's enunciate through an example.

\subsection{Example from 'Entropy Demystified'}

Suppose there are three dice, and we are asked to bet on the sum of the outcomes of tossing the three dice simultaneously. We will not consider betting on 3 and 18 as both 3 and 18 have only one way of occurring; $1: 1: 1$ or $6: 6: 6$, respectively, and we intuitively judge that these events are relatively rare.7, is a better choice because there are more partitions of the number 7 into three numbers (between 1 and 6), that is, 7 can be obtained as a result of four possible partitions: 1:1:5, 1:2:4, 1:3:3, 2:2:3. Using a simple probabilistic analysis it can be shown that the relative chances of winning with 9 and 10 , is $25: 27$, that is, favouring the choice of 10 . Thus, the best choice of a winning number, 10. But what does it mean that 10 is the "best" choice and that this is the 
"correct" winning number? I could choose 10 and you could choose 3 and you might win the game. Does our calculation guarantee that if I choose10, I will always win? No, so what does the ratio 25:27 mean? We use the theory of probability to give us an answer. It says that "if we play this game many times (Therefore, clearly pointing towards the arrow of time), the probability that the choice of 9 will win is $25 / 216$, whereas the probability that the choice of 10 will win is slightly larger, 27/216."

Therefore, every system proceeds to the most probable state or stable state over time is a postulate buried in probability theory upon which proposed law is based. Since this process of system proceeding to stable state happens over time. The arrow of time does exist and it points towards the most probable state.

\section{A case for a "Theory of Everything"}

Theory of Everything is the ultimate theory sought after by physicists and scientific philosophers. It is the description of reality that can be enunciated by a single framework. Stephan Hawking [23] realized that there could be more than one theory of everything each theory treating the universe in its own fishbowl. This article claims that the framework proposed in this paper is one of "Theory of Everything". This seems appropriate as all the physical models that can be encapsulated by the proposed framework. Every model that represents reality can be thought to consist of systems that encapsulate certain systems and are encapsulated by other systems; State variables encapsulate the information that is to be considered. The proposed law predicts the evolution of the variables. Hence, this is a fundamental law valid to all real models. Some may argue this law is a Kantian "a priori" whose nature is one, that of, empirical universalism - an arbitrary extension of validity or a kind of a truism. But, the proposed theory does offer very important insights. First, it resolves the ancient mystery of entropy. Chaos in the Universe does not" always" increase as the order is more likely to increase than chaos. Throw a ball in a bowl it will come to rest. Hence, Order is the ultimate 
desire. Moreover, unlike most sought after "Theory of Everything's" such as String theory which currently has no metaphysical interpretation, the proposed theory is not just physical it applies to the mind as well.

\section{Conclusions}

This article reproaches the idea - Chaos always increases, instead, it is order that increases and presents Entropy as a quantity that encapsulates different quantities such as Velocity, Pressure, Energy and so on, therefore entropy is essentially information about the state of the system and is stored in form of mathematical abstractions and evolution is governed by Second law which is akin Hegel's philosophy and also gives an explanation for time's arrow, resolving Loschmidt's paradox.

For a mathematical framework, the Boltzmann's equation, definition by Claude Shannon and papers by authors such as Elliot Leib [24] [25] could suffice. Although the author feels, if one is optimistic about a better mathematical framework then it is an open problem.

Let us conclude by a quotation from Solomon, "In a world that has come to see ideas and collective enthusiasm with horror, Hegel becomes a gateway to a new world, where ideas are the key to consciousness, where the philosopher becomes the spokesman for the times and the prophet of a united humanity. It is a world in which archaic terms like "harmony" and "humanity" still makes sense - indeed, still, give us something to hope for. It is a world worth, at least, considering".

\section{References}

1. Schneider, E. D. \& Kay, J. J. 1994. Life as a manifestation of the second law of thermodynamics, Mathematical and Computer Modelling

2. Ben-Naim, A., 2017. Can entropy be defined for and the Second Law applied to the entireuniverse?,ArXiv:1705.01100 [Cond-Mat, Physics: Physics] 
3. Brissaud, J.-B. 2005. The meanings of entropy, Entropy.

4. Shannon, C. E., 1948. A Mathematical Theory of Communication, Bell System Technical Journal.

5. Brillouin, L., 1951. Maxwell's demon cannot operate: Information and entropy. I. Journal of Applied Physics, 22(3), pp.334-337.

6. Brillouin, L., 1951. Physical entropy and information. II. Journal of Applied Physics, 22(3), pp.338343.

7. Avery, J. S. , 2012. Information Theory and Evolution, WORLD SCIENTIFIC,

8. Rovelli, C., 2019. The order of time. Riverhead books.

9. Rovelli, C., 2018. Reality is not what it seems: The journey to quantum gravity. Penguin.

10. Ben-Naim, A. 2017. The Four Laws That Do Not Drive The Universe: Elements of Thermodynamics for the Curious and Intelligent, WORLD SCIENTIFIC,

11. Ben-Naim, A. 2007. Entropy Demystified: The Second Law Reduced to Plain Common Sense, WORLD SCIENTIFIC

12. Solomon, R.C., 1985. In the spirit of Hegel. Oxford University Press.

13. Aerts, D., de Bianchi, M. S., Sozzo, S., \& Veloz, T. (2018). On the conceptuality interpretation of quantum and relativity theories. Foundations of Science, 1-50.

14. Aerts, D. (2018). Relativity theory refounded. Foundations of science, 23(3), 511-547.

15. Hegel, G.W.F., 1998. Phenomenology of spirit. Motilal Banarsidass Publ..

16. Bowman, G. E. 2008. Essential quantum mechanics Oxford: Oxford Univ. Press. 
17. Zermelo, E., 2010. Ernst Zermelo-Collected Works/Gesammelte Werke: Volume I/Band I-Set Theory, Miscellanea/Mengenlehre, Varia (Vol. 21). Springer Science \& Business Media.

18. Cohen R.S., Stachel J.J. ,1979. Max Planck and the Statistical Definition of Entropy [1959b].

In: Cohen R.S., Stachel J.J. (eds) Selected Papers of Léon Rosenfeld. Boston Studies in the Philosophy of Science, vol 21. Springer, Dordrecht

19. Planck, M., 1901. On the law of distribution of energy in the normal spectrum. Annalen der physik, 4(553), p.1.

20. Lacan, J., 1998. The four fundamental concepts of psycho-analysis (Vol. 11). WW norton \& Company.

21. Žižek, S., 2014. The most sublime hysteric: Hegel with Lacan. John Wiley \& Sons.

22. Rothstein, J., 1974. Loschmidt's and Zermelo's paradoxes do not exist. Foundations of Physics, 4(1), pp.83-89.

23. Hawking, S. \& Mlodinow, L. 2010. The (Elusive) Theory of Everything. Scientific American,303(4), $68-71$.

24. Lieb, E. H. \& Yngvason, J. 2004. A Fresh Look at Entropy and the Second Law of Thermodynamics. In B. Nachtergaele, J. P. Solovej, \& J. Yngvason (Eds.), Statistical Mechanics: Selecta of Elliott H. Lieb (pp. 365-370). Berlin, Heidelberg: Springer Berlin Heidelberg.

25. Lieb, E. H. \& Yngvason, J. 2004. A Guide to Entropy and the Second Law of Thermodynamics. In B. Nachtergaele, J. P. Solovej, \& J. Yngvason (Eds.), Statistical Mechanics: Selecta of Elliott H. Lieb (pp. 353-363). Berlin, Heidelberg: Springer Berlin Heidelberg. 14. Lieb, E. H., \& Yngvason, J. 2004 The Mathematical Structure of the Second Law of Thermodynamics. ArXiv:Math-Ph/0204007. 
